\title{
Complete genome sequence of Spirosoma linguale type strain $\left(1^{\mathrm{T}}\right)$
}

\author{
Kathleen Lail ${ }^{1}$, Johannes Sikorski ${ }^{2}$, Elizabeth Saunders ${ }^{3}$, Alla Lapidus ${ }^{1}$, Tijana Glavina Del \\ Rio $^{1}$, Alex Copeland ${ }^{1}$, Hope Tice ${ }^{1}$, Jan-Fang Cheng ${ }^{1}$, Susan Lucas ${ }^{1}$, Matt Nolan ${ }^{1}$, David \\ Bruce $^{1,3}$, Lynne Goodwin ${ }^{1,3}$, Sam Pitluck ${ }^{1}$, Natalia Ivanova ${ }^{1}$, Konstantinos Mavromatis ${ }^{1}$, \\ Galina Ovchinnikova ${ }^{1}$, Amrita Pati ${ }^{1}$, Amy Chen ${ }^{4}$, Krishna Palaniappan ${ }^{4}$, Miriam Land $^{1,5}$, \\ Loren Hauser ${ }^{1,5}$, Yun-Juan Chang ${ }^{1,5}$, Cynthia D. Jeffries ${ }^{1,5}$, Patrick Chain ${ }^{1,6}$, Thomas Brettin ${ }^{1,3}$, \\ John C. Detter ${ }^{1,3}$, Andrea Schütze ${ }^{2}$, Manfred Rohde ${ }^{7}$, Brian J. Tindall ${ }^{2}$, Markus Göker ${ }^{2}$, Jim \\ Bristow ${ }^{1}$, Jonathan A. Eisen ${ }^{1,8}$, Victor Markowitz ${ }^{4}$, Philip Hugenholtz ${ }^{1}$, Nikos C. Kyrpides ${ }^{1 *}$, \\ Hans-Peter Klenk ${ }^{2}$, and Feng Chen ${ }^{1}$ \\ ${ }^{1}$ DOE Joint Genome Institute, Walnut Creek, California, USA \\ ${ }^{2}$ DSMZ - German Collection of Microorganisms and Cell Cultures GmbH, Braunschweig, \\ Germany \\ ${ }^{3}$ Los Alamos National Laboratory, Bioscience Division, Los Alamos, New Mexico, USA \\ ${ }^{4}$ Biological Data Management and Technology Center, Lawrence Berkeley National \\ Laboratory, Berkeley, California, USA \\ ${ }^{5}$ Oak Ridge National Laboratory, Oak Ridge, Tennessee, USA \\ ${ }^{6}$ Lawrence Livermore National Laboratory, Livermore, California, USA \\ ${ }^{7}$ HZI - Helmholtz Centre for Infection Research, Braunschweig, Germany \\ ${ }^{8}$ University of California Davis Genome Center, Davis, California, USA \\ ${ }^{*}$ Corresponding author: Nikos C. Kyrpides
}

Keywords: psychroactive, oligotrophic, aerobic, ringlike morphology, non-pathogenic, freeliving, Cytophagaceae, GEBA

\begin{abstract}
Spirosoma linguale Migula 1894 is the type species of the genus. S. linguale is a free-living and non-pathogenic organism, known for its peculiar ringlike and horseshoe-shaped cell morphology. Here we describe the features of this organism, together with the complete genome sequence and annotation. This is only the third completed genome sequence of a member of the family Cytophagaceae. The 8,491,258 bp long genome with its eight plasmids, 7,069 protein-coding and 60 RNA genes is part of the Genomic Encyclopedia of Bacteria and Archaea project.
\end{abstract}

\section{Introduction}

Strain $1^{\mathrm{T}}$ (= DSM 74 = ATCC 33905 = LMG 10896) is the type strain of the species Spirosoma linguale, which is the type species of the genus Spirosoma. The genus currently consists of five species [1]. Strain $1^{\mathrm{T}}$ is reported to be isolated from a laboratory water bath (websites of DSMZ and ATCC), however, a proper reference could not be identified. Another strain of $S$. linguale was isolated from fresh water from deep wells in Long Beach, California, USA [2]. Other strains from the genus Spirosoma were isolated from high arctic permafrost soil in Norway [3], soil from a ginseng field in Pocheon province, South Korea [4], and fresh water from the Woopo wetlands, South Korea [5]. This would allow the hypothesis that $S$. linguale is a free-living species with a worldwide distribution. The genus name Spirosoma derives from 'spira' from Latin meaning coil combined with 'soma', Latin for 'body', resulting in 'coiled body' [1]. Spirosoma was the first genus in the family Spirillaceae in Migula's "System der Bakterien" [6]. The species name is effectively published by Migula in 1894 [7] and validly published by Skerman in 1980 [8]. Various taxonomic treatments have placed this organism either in the family "Flexibacteraceae" or the family Cyto- 
phagaceae. This would appear to be due to a number of nomenclatural problems. The family "Flexibacteriaceae" as outlined in TOBA 7.7 would include Cytophaga hutchinsonii, which is the type species of the genus Cytophaga, which, in turn is the type of the family Cytophagaceae, a name that may not be replaced by the family name "Flexibacteriaceae" as long as Cytophaga hutchinsonii is one of the included species. However, the topology of the 16S rDNA based dendrogram indicates that it may be possible to define a second family, including the genus Spirosoma, but excluding Cytophaga hutchinsonii. At the same time, the family Cytophagaceae may be defined to exclude the type species of the genus Flexibacter and members of the genus Spirosoma. It should also be remembered that the genus Spirosoma is the type of the family Spirosomaceae Larkin and Borrall 1978. At present the higher taxonomic ranks of this group of organisms lacks formal modern descriptions and circumscriptions making it difficult to make definitive state- ments that would hold over the next few years. Here we present a summary classification and a set of features for $S$. linguale $1^{\mathrm{T}}$, together with the description of the complete genomic sequencing and annotation.

\section{Classification and features}

Uncultured clone sequences in Genbank showed $96 \%$ or less sequence identity to the $16 \mathrm{~S}$ gene sequence (AM000023) of strain S. linguale $1^{\mathrm{T}}$. No reasonable sequence similarity $(>87 \%)$ to any metagenomic survey were reported from the NCBI BLAST server (October 2009).

Figure 1 shows the phylogenetic neighborhood of for $S$. linguale $1^{\mathrm{T}}$ in a $16 \mathrm{~S}$ rRNA based tree. The sequences of the four identical 16S rRNA gene copies in the genome of $S$. linguale $1^{\mathrm{T}}$ are also identical with the previously published $16 \mathrm{~S}$ rRNA sequence generated from LMG 10896 (AM000023).

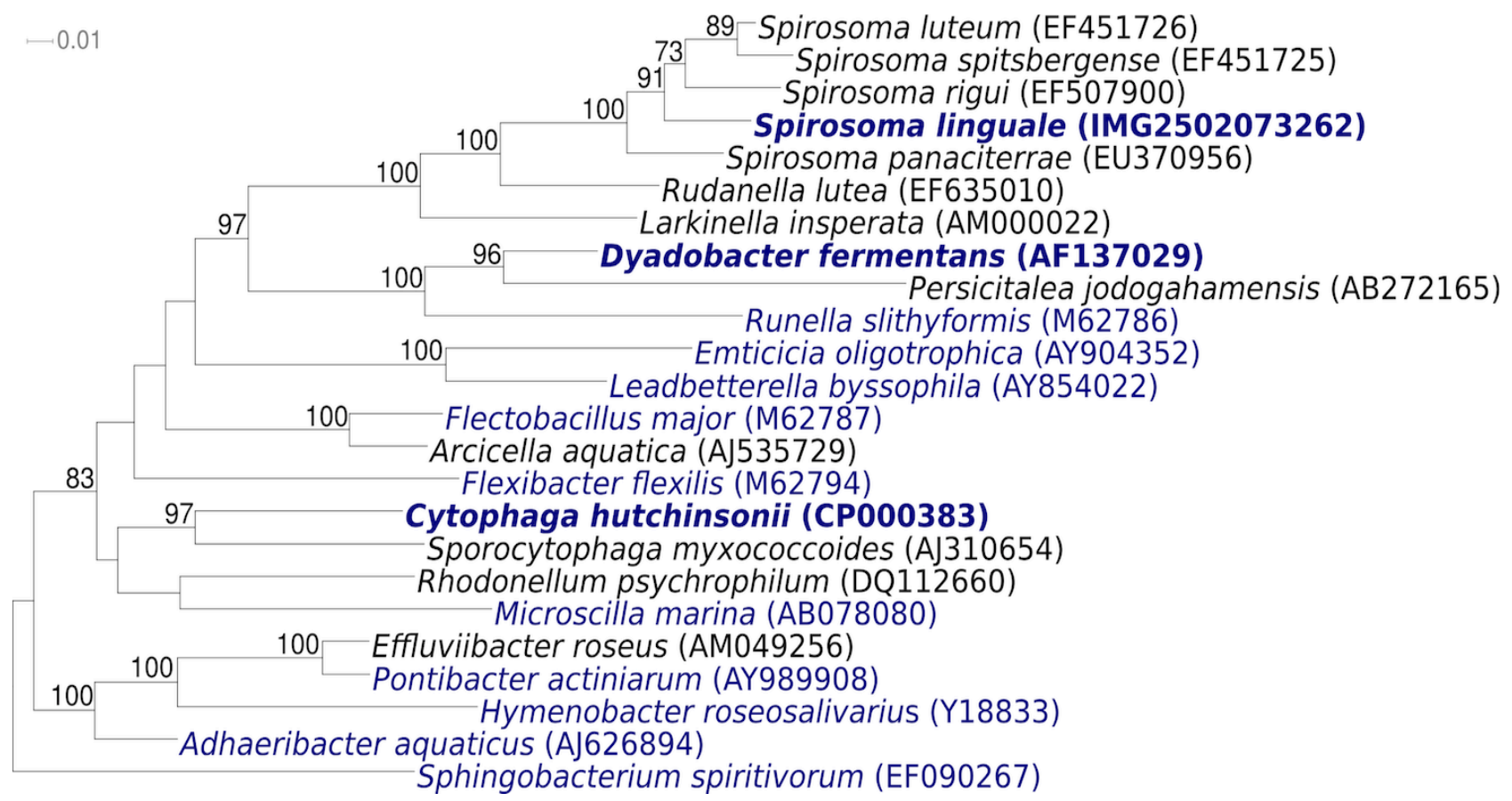

Figure 1. Phylogenetic tree highlighting the position of $S$. linguale $1^{\top}$ and the type strains of the other species within the genus relative to the other type strains within the family Cytophagaceae. The tree was inferred from 1,320 aligned characters [9,10] of the 16S rRNA gene sequence under the maximum likelihood criterion [11] and rooted with the type strain of the family Sphingobacteriaceae. The branches are scaled in terms of the expected number of substitutions per site. Numbers above branches are support values from 1,000 bootstrap replicates if larger than 60\%. Lineages with type strain genome sequencing projects registered in GOLD [12] are shown in blue, published genomes such as the one of Dyadobacter fermentans [13] in bold. 
On TGEY medium [14], strain S. linguale $1^{\mathrm{T}}$ forms mucoid, opaque, and smooth colonies with a yellowish nondiffusible pigment [15]. The colony size is $2-4 \mathrm{~mm}$, circular, with entire margins and convex elevation. In broth, growth is aerobic (Table 1) with even turbidity and flaky sediment [15]. The Gram-negative cells have round ends and show vibroid, horseshoe, and ring-like shapes, as well as coils and spiral forms [Figure 2 and ref. 15]. The cell width is $0.5-1.0 \mu \mathrm{m}$, and the outer ring diameter is $1.5-3.0 \mu \mathrm{m}$. The cell length is $2.0-5.0 \mu \mathrm{m}$ [22]. Reports on filaments are conflicting [15,22].

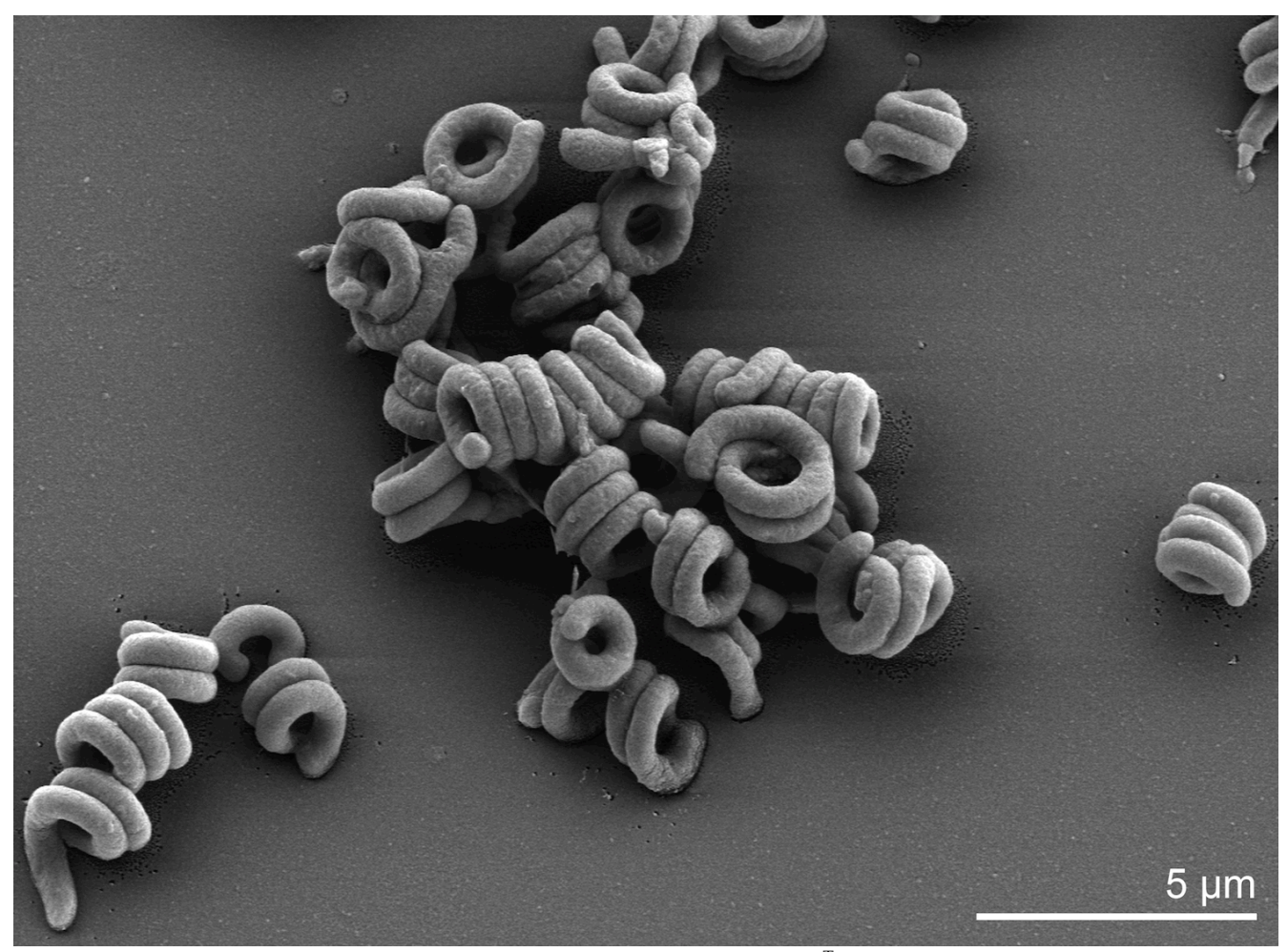

Figure 2. Scanning electron micrograph of S. linguale $1^{\top}$

Strain S. linguale $1^{\mathrm{T}}$ produces oxidatively acid from arabinose, ribose, xylose, rhamnose, fructose, galactose, glucose, mannose, $\alpha$-methyl-D-glucoside, salicin, cellobiose, lactose, maltose, melibiose, sucrose, trehalose, raffinose, dextrin and inulin, but not from sorbose, glycerol, erythritol, dulcitol, mannitol, and sorbitol [22]. On the enzymatic level, strain S. linguale $1^{\mathrm{T}}$ is positive for oxidase, catalase, ONPGreaction, and, albeit weakly, for phosphatase, but negative for urease, lecithinase, lysine decarboxylase, phenylalanine deaminase, and hemolysin, indole, methyl red, Voges-Proskauer, $\mathrm{NO}_{3}$ reduction and $\mathrm{H}_{2} \mathrm{~S}$ reactions [22]. Strain $S$. linguale $1^{\mathrm{T}}$ hydrolyzes esculin, tributyrin, gelatin, and, less well, starch and casein, but not cellulose and chitin [22]. It utilizes for growth on basal medium [25] glycerol phosphate, succinate, tartrate, and malonate as single carbon source, but not acetate, benzoate, citrate, formate, methylamine, propionate, and methanol
[22]. Strain $S$. linguale $1^{\mathrm{T}}$ grows well on nutrient agar, nutrient agar $+5 \%$ sucrose, MicrocyclusSpirosoma agar, and yeast extract tryptone agar, weakly on peptonized milk agar, blood, and chocolate, and not on eosin methylene blue agar, phenol red mannitol salt agar, phenyl ethyl alcohol agar, trypticase soy agar (TSA), TSA + 3\% glucose, TSA + $3 \%$ sucrose, McConkey, bismuth sulfide agar, and Salmonella-Shigella agar [22]. Strain S. linguale $1^{\mathrm{T}}$ is susceptible to actinomycin D $(100 \mu \mathrm{g} / \mathrm{ml})$, ampicillin $(10 \mu \mathrm{g})$, aureomycin $(15 \mu \mathrm{g})$, carbenicillin (50 $\mu \mathrm{g})$, erythromycin $(15 \mu \mathrm{g})$, furadantin/macrodantin $(300 \mu \mathrm{g})$, gentamicin $(10 \mu \mathrm{g})$, kanamycin $(30 \mu \mathrm{g})$, mitomycin $\mathrm{C}(1 \mu \mathrm{g} / \mathrm{ml})$, neomycin $(30 \mu \mathrm{g})$, penicillin $G$ (10 units), streptomycin (10 $\mu \mathrm{g})$, sulfamethoxyzole/trimethopterin $(25 \mu \mathrm{g})$, sulfathiazole $(300 \mu \mathrm{g})$, and tetracycline $(30 \mu \mathrm{g})$, but resistant to colistin $(10 \mu \mathrm{g})$, polymixin B (300 units), and triple sulfa (1 mg) [22]. 
Table 1. Classification and general features of $S$. linguale $1^{\top}$ according to the MIGS recommendations [16]

\begin{tabular}{|c|c|c|c|}
\hline MIGS ID & Property & Term & Evidence code \\
\hline & \multirow{8}{*}{ Current classification } & Domain Bacteria & TAS [17] \\
\hline & & Phylum Bacteroidetes & TAS $[18,19]$ \\
\hline & & Class Sphingobacteria & TAS $[18,20]$ \\
\hline & & Order Sphingobacteriales & TAS $[18,20]$ \\
\hline & & Family Cytophagaceae & TAS $[8,21]$ \\
\hline & & Genus Spirosoma & TAS $[3,7,8]$ \\
\hline & & Species Spirosoma linguale & TAS [7] \\
\hline & & Type strain 1 & TAS $[7,8]$ \\
\hline & Gram stain & negative & TAS [15] \\
\hline & Cell shape & $\begin{array}{l}\text { vibroid, horseshoe, and ring-like shapes; } \\
\text { spiral form }\end{array}$ & TAS [15] \\
\hline & Motility & non-motile & TAS [15] \\
\hline & Sporulation & nonsporulating & NAS \\
\hline & Temperature range & $5^{\circ} \mathrm{C}-39^{\circ} \mathrm{C}$ & TAS [5] \\
\hline & Optimum temperature & $20^{\circ} \mathrm{C}-30^{\circ} \mathrm{C}$ & TAS [5] \\
\hline & Salinity & $0-1.25 \%(w / v)$ & TAS [5] \\
\hline \multirow[t]{3}{*}{ MIGS-22 } & Oxygen requirement & aerobic & TAS [15] \\
\hline & Carbon source & $\begin{array}{l}\text { glycerol phosphate, succinate, tartrate, } \\
\text { malonate }\end{array}$ & TAS [22] \\
\hline & Energy source & carbohydrates & TAS [22] \\
\hline MIGS-6 & Habitat & Laboratory water bath & NAS \\
\hline MIGS-15 & Biotic relationship & free-living & NAS \\
\hline \multirow[t]{3}{*}{ MIGS-14 } & Pathogenicity & not reported & NAS \\
\hline & Biosafety level & 1 & TAS [23] \\
\hline & Isolation & not reported & NAS \\
\hline MIGS-4 & Geographic location & Germany & NAS \\
\hline MIGS-5 & Sample collection time & not reported & \\
\hline $\begin{array}{l}\text { MIGS-4.1 } \\
\text { MIGS-4.2 }\end{array}$ & $\begin{array}{l}\text { Latitude } \\
\text { Longitude }\end{array}$ & not reported & \\
\hline MIGS-4.3 & Depth & not reported & \\
\hline MIGS-4.4 & Altitude & not reported & \\
\hline
\end{tabular}

Evidence codes - IDA: Inferred from Direct Assay (first time in publication); TAS: Traceable Author Statement (i.e., a direct report exists in the literature); NAS: Non-traceable Author Statement (i.e., not directly observed for the living, isolated sample, but based on a generally accepted property for the species, or anecdotal evidence). These evidence codes are from of the Gene Ontology project [24]. If the evidence code is IDA, then the property was directly observed for a live isolate by one of the authors, or an expert mentioned in the acknowledgements.

\section{Chemotaxonomy}

Earlier studies report $\mathrm{C}_{16: 1}$ to be the dominant fatty acid (47.9\%), followed by iso- $\mathrm{C}_{17: 0}(20.1 \%), \mathrm{C}_{16: 0}$ $(14.2 \%)$, iso- $\mathrm{C}_{15: 0}(11.0 \%)$ and iso- $\mathrm{C}_{13: 0}(3.4 \%)$. Anteiso and hydroxy fatty acids are each below $2.1 \%$ [26]. The fatty acids comprise a complex mixture of straight chain saturated and unsaturated fatty acids, together with iso-branched and 3 -hydroxylated iso-branched fatty acids. The fatty acids comprise iso- $\mathrm{C}_{13: 0}$ (2.2\%), iso- $\mathrm{C}_{15: 0}(9.3 \%)$, iso- $\mathrm{C}_{15: 0} 3-\mathrm{OH}$ (3.4\%), anteiso- $\mathrm{C}_{15: 0}(2.6 \%), \mathrm{C}_{16: 0}$ (3.6\%), $\mathrm{C}_{16: 0} 3-\mathrm{OH}(2.2 \%), \mathrm{C}_{16: 1} \omega \mathrm{cc}(22.2 \%), \mathrm{C}_{17: 0}$ $2-\mathrm{OH} \quad(1.0 \%)$, iso- $\mathrm{C}_{17: 0} 3-\mathrm{OH} \quad(8.6 \%)$, iso- $\mathrm{C}_{13: 0}$ (2.2\%), $\mathrm{C}_{17: 1} \omega 9 \mathrm{c}(1.2 \%)$ and $\mathrm{C}_{16: 1} \omega \mathrm{7c}$ and/or iso- $\mathrm{C}_{15: 0} 2-\mathrm{OH}$ (42.4\%). The polar lipids comprise phosphatidylethanolamine and a number of lipids and amino lipids that were not further characte- 
rized. The fatty acid pattern is typical of the evolutionary group currently defined as the phylum Bacteroidetes. Furthermore the presence of phosphatidylethanolamine as the predominant/sole diglyceride based phospholipid is also typical of the vast majority of the phylum Bacteroidetes. Limited detailed studies indicate that this phospholipid contains both saturated and unsaturated straight chain fatty acids. Hydroxylated fatty acids are not present in this compound. In contrast, the limited studies on the amino lipids of Flavobacterium johnsoniae indicate that they are amino acid based, with a 3-OH fatty acid in amide linkage with a free amino group of the amino acid. The 3$\mathrm{OH}$ fatty acid is further esterified with either a non-hydroxylated fatty acid, or with a second hydroxylated fatty acid. The presence of lipids that did not stain further running in proximity with the major aminolipid may also be indicative of capnines. The failure to resolve the fatty acids reported by the MIDI Sherlock MIS system as "summed feature 4" $\mathrm{C}_{16: 1 \omega 7 \mathrm{c}} /$ iso $\mathrm{C}_{15: 0} 2-\mathrm{OH}$ is problematic for genomics, since it either indicates that two mechanisms of introducing double bonds into fatty acids are present $\left(\mathrm{C}_{16: 1 \omega 5 \mathrm{c}}\right.$ and $\left.\mathrm{C}_{16: 1 \omega 7 \mathrm{c}}\right)$ or a fatty acid 2-hydroxylase is present. Furthermore, the distribution of $3-\mathrm{OH}$ and $2-\mathrm{OH}$ fatty acids among the amino- and non-staining lipids may also be characteristic. The main isoprenoid quinone is MK-7 (91.5\%), followed by MK-8 (7.2\%) and MK-6 (1.3\%) [26].

\section{Genome sequencing and annotation Genome project history}

This organism was selected for sequencing on the basis of its phylogenetic position, and is part of the Genomic Encyclopedia of Bacteria and Archaea project. The genome project is deposited in the Genome OnLine Database [12] and the complete genome sequence is deposited in GenBank. Sequencing, finishing and annotation were performed by the DOE Joint Genome Institute (JGI). A summary of the project information is shown in Table 2.

Table 2. Genome sequencing project information

\begin{tabular}{|c|c|c|}
\hline MIGS ID & Property & Term \\
\hline MIGS-31 & Finishing quality & Finished \\
\hline \multirow[b]{2}{*}{ MIGS-28 } & \multirow[b]{2}{*}{ Libraries used } & $\begin{array}{l}\text { Two Sanger libraries: } 8 \mathrm{~kb} \text { pMCL200 } \\
\text { and fosmid pcc1Fos }\end{array}$ \\
\hline & & $\begin{array}{l}\text { One } 454 \text { pyrosequence and one } \\
\text { Solexa standard library }\end{array}$ \\
\hline MIGS-29 & Sequencing platforms & ABI3730, 454 GS FLX, Illumina GA \\
\hline MIGS-31.2 & Sequencing coverage & $10.1 \times$ Sanger; $18.4 \times$ pyrosequence \\
\hline MIGS-30 & Assemblers & Newbler 1.1.02.15, phrap \\
\hline \multirow[t]{6}{*}{ MIGS-32 } & Gene calling method & Prodigal, GenePRIMP \\
\hline & INSDC ID & $\begin{array}{l}\text { CP001769 (chromosome) } \\
\text { CP001770-77 (plasmids) }\end{array}$ \\
\hline & Genbank Date of Release & January 13, 2010 \\
\hline & GOLD ID & Gc01186 \\
\hline & NCBI project ID & 28817 \\
\hline & Database: IMG-GEBA & 2501939635 \\
\hline \multirow[t]{2}{*}{ MIGS-13 } & Source material identifier & DSM 74 \\
\hline & Project relevance & Tree of Life, GEBA \\
\hline
\end{tabular}

\section{Growth conditions and DNA isolation}

S. linguale $1^{\mathrm{T}}$, DSM 74, was grown in DSMZ medium 7 [27] at $28^{\circ} \mathrm{C}$. DNA was isolated from 0.5$1 \mathrm{~g}$ of cell paste using Qiagen Genomic 500 DNA Kit (Qiagen, Hilden, Germany) with cell lysis modification st/L [28] and one hour incubation at $37^{\circ} \mathrm{C}$.

\section{Genome sequencing and assembly}

The genome was sequenced using a combination of Sanger and 454 sequencing platforms. All general aspects of library construction and sequencing can be found at http://www.jgi.doe.gov/. 454 Pyrosequencing reads were assembled using the Newbler assembler version 1.1.02.15 (Roche). 
Large Newbler contigs were broken into 9,401 overlapping fragments of $1,000 \mathrm{bp}$ and entered into assembly as pseudo-reads. The sequences were assigned quality scores based on Newbler consensus q-scores with modifications to account for overlap redundancy and to adjust inflated qscores. A hybrid 454/Sanger assembly was made using the parallel phrap assembler (High Performance Software, LLC). Possible mis-assemblies were corrected with Dupfinisher [29] or transposon bombing of bridging clones (Epicentre Biotechnologies, Madison, WI). Gaps between contigs were closed by editing in Consed, custom primer walk or PCR amplification. A total of 974 Sanger finishing reads were produced to close gaps, to resolve repetitive regions, and to raise the quality of the finished sequence. Illumina reads were used to improve the final consensus quality using an inhouse developed tool (the Polisher). The error rate of the completed genome sequence is less than 1 in 100,000. Together all sequence types provided $28.5 \times$ coverage of the genome. The final assembly contains 87,186 Sanger and 666,973 pyrosequence reads.

\section{Genome annotation}

Genes were identified using Prodigal [30] as part of the Oak Ridge National Laboratory genome annotation pipeline, followed by a round of manual curation using the JGI GenePRIMP pipeline [31]. The predicted CDSs were translated and used to search the National Center for Biotechnology Information (NCBI) nonredundant database, UniProt, TIGRFam, Pfam, PRIAM, KEGG, COG, and InterPro databases. Additional gene prediction analysis and manual functional annotation was performed within the Integrated Microbial Genomes Expert Review (IMG-ER) platform [32].

\section{Genome properties}

The genome consists of a 8,078,757 bp long chromosome and eight plasmids with 6,072 to 189,452 bp length (Table 3 and Figure 3). Of the 7,129 genes predicted, 7,069 were proteincoding genes, and 60 RNAs; 131 pseudogenes were also identified. The majority of the proteincoding genes $(61.5 \%)$ were assigned with a putative function while those remaining were annotated as hypothetical proteins. The distribution of genes into COGs functional categories is presented in Table 4.

Table 3. Genome Statistics

\begin{tabular}{lrr}
\hline Attribute & \multicolumn{1}{c}{ Value } & \% of Total \\
\hline Genome size (bp) & $8,491,258$ & $100.00 \%$ \\
DNA coding region (bp) & $7,518,086$ & $88.54 \%$ \\
DNA G+C content (bp) & $4,258,276$ & $50.15 \%$ \\
Number of replicons & 9 & \\
Extrachromosomal elements & 8 & \\
Total genes & 7,129 & $100.00 \%$ \\
RNA genes & 60 & $0.84 \%$ \\
rRNA operons & 4 & \\
Protein-coding genes & 7,069 & $99.16 \%$ \\
Pseudo genes & 131 & $1.84 \%$ \\
Genes with function prediction & 4,386 & $61.52 \%$ \\
Genes in paralog clusters & 1,713 & $2.71 \%$ \\
Genes assigned to COGs & 4,306 & $60.40 \%$ \\
Genes assigned Pfam domains & 4,519 & $63.39 \%$ \\
Genes with signal peptides & 2,271 & $41.86 \%$ \\
Genes with transmembrane helices & 1,606 & $22.53 \%$ \\
CRISPR repeats & 2 & \\
\hline
\end{tabular}



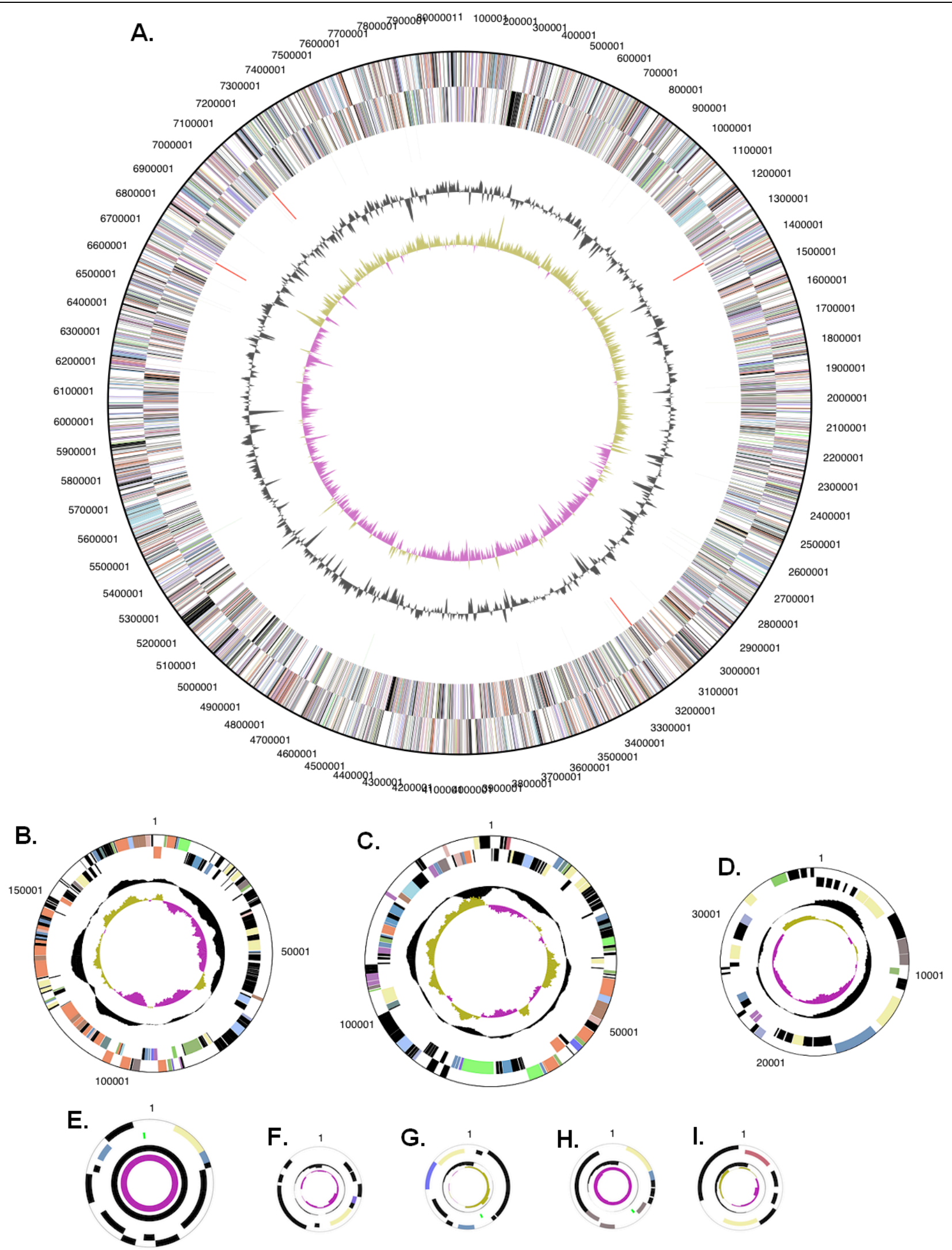

Figure 3. Graphical circular map of the chromosome (A) and the eight plasmids: pSLIN01 (B), pSLIN02 (C), pSLIN03 (D), pSLIN04 (E), pSLIN05 (Fs), pSLIN06 (G), pSLIN07 (H), pSLIN08 (I). Plasmids not drawn to scale. From outside to the center: Genes on forward strand (color by COG categories), Genes on reverse strand (color by COG categories), RNA genes (tRNAs green, rRNAs red, other RNAs black), GC content, GC skew. 
Table 4. Number of genes associated with the general COG functional categories

\begin{tabular}{crrl}
\hline Code & Value & \%age & Description \\
\hline J & 173 & 2.4 & Translation, ribosomal structure and biogenesis \\
A & 1 & 0.0 & RNA processing and modification \\
K & 398 & 6.6 & Transcription \\
L & 251 & 3.6 & Replication, recombination and repair \\
B & 1 & 0.0 & Chromatin structure and dynamics \\
D & 32 & 0.5 & Cell cycle control, mitosis and meiosis \\
Y & 0 & 0.0 & Nuclear structure \\
V & 161 & 2.3 & Defense mechanisms \\
T & 380 & 5.4 & Signal transduction mechanisms \\
M & 411 & 5.8 & Cell wall/membrane biogenesis \\
N & 14 & 0.2 & Cell motility \\
Z & 0 & 0.0 & Cytoskeleton \\
W & 0 & 0.0 & Extracellular structures \\
U & 68 & 1.0 & Intracellular trafficking and secretion \\
O & 161 & 2.3 & Posttranslational modification, protein turnover, chaperones \\
C & 223 & 3.2 & Energy production and conversion \\
G & 383 & 5.4 & Carbohydrate transport and metabolism \\
E & 297 & 4.2 & Amino acid transport and metabolism \\
F & 80 & 1.1 & Nucleotide transport and metabolism \\
H & 174 & 2.5 & Coenzyme transport and metabolism \\
I & 162 & 2.3 & Lipid transport and metabolism \\
P & 277 & 3.9 & Inorganic ion transport and metabolism \\
Q & 121 & 1.7 & Secondary metabolites biosynthesis, transport and catabolism \\
R & 611 & 8.6 & General function prediction only \\
S & 424 & 6.0 & Function unknown \\
- & 2,823 & 39.9 & Not in COGs \\
\hline
\end{tabular}

\section{Acknowledgements}

We would like to gratefully acknowledge the help of Susanne Schneider (DSMZ) for DNA extraction and quality analysis. This work was performed under the auspices of the US Department of Energy's Office of Science, Biological and Environmental Research Program, and by the University of California, Lawrence Berkeley National Laboratory under contract No. DE-

\section{References}

1. Euzéby JP. List of bacterial names with standing in nomenclature: A folder available on the Internet. Int J Syst Bacteriol 1997; 47:590-592. PubMed doi:10.1099/00207713-47-2-590

2. Raj HD. A new species - Microcyclus flavus. Int J Syst Bacteriol 1970; 20:61-81. doi:10.1099/00207713-20-1-61

3. Finster KW, Herbert RA, Lomstein BA. Spirosoma spitsbergense sp. nov. and Spirosoma luteum sp. nov., isolated from a high Arctic permafrost soil, and emended description of the genus Spirosoma.
AC02-05CH11231, Lawrence Livermore National Laboratory under Contract No. DE-AC52-07NA27344, Los Alamos National Laboratory under contract No. DEAC02-06NA25396, and Oak Ridge National Laboratory under contract DE-AC05-000R22725, as well as German Research Foundation (DFG) INST 599/1-1 and SI 1352/1-2.

Int J Syst Evol Microbiol 2009; 59:839-844. PubMed doi:10.1099/ijs.0.002725-0

4. Ten LN, Xu JL, Jin FX, Im WT, Oh HM, Lee ST. Spirosoma panaciterrae sp. nov., isolated from soil. Int J Syst Evol Microbiol 2009; 59:331-335. PubMed doi:10.1099/ijs.0.002436-0

5. Baik KS, Kim MS, Park SC, Lee DW, Lee SD, Ka JO, Choi SK, Seong CN. Spirosoma rigui sp. nov., isolated from fresh water. Int I Syst Evol Microbiol 2007; 57:2870-2873. PubMed doi:10.1099/ijs.0.65302-0 
6. Migula W. 1900. System der Bakterien, Vol 2. Gustav Fischer, Jena.

7. Migula W. Über ein neues System der Bakterien. Arb Bakteriol Inst Karlsruhe 1894; 1:235-238.

8. Skerman VBD, McGowan V, Sneath PHA. Approved Lists of Bacterial Names. Int J Syst Bacteriol 1980; 30:225-420. doi:10.1099/00207713$\underline{30-1-225}$

9. Castresana J. Selection of conserved blocks from multiple alignments for their use in phylogenetic analysis. Mol Biol Evol 2000; 17:540-552. PubMed

10. Lee C, Grasso C, Sharlow MF. Multiple sequence alignment using partial order graphs. Bioinformatics 2002; 18:452-464. PubMed doi:10.1093/bioinformatics/18.3.452

11. Stamatakis A, Hoover P, Rougemont J. A Rapid Bootstrap Algorithm for the RAxML Web Servers. Syst Biol 2008; 57:758-771. PubMed doi:10.1080/10635150802429642

12. Liolios K, Chen IM, Mavromatis K, Tavernarakis N, Hugenholtz P, Markowitz VM, Kyrpides NC. The Genomes On Line Database (GOLD) in 2009: status of genomic and metagenomic projects and their associated metadata. Nucleic Acids Res 2010; 38:D346-D354. PubMed doi:10.1093/nar/gkp848

13. Lang E, Lapidus A, Chertkov O, Brettin T, Detter JC, Han C, Copeland A, Glavina Del Rio T, Nolan $\mathrm{M}$, Chen $\mathrm{F}$, et al. Complete genome sequence of Dyadobacter fermentans type strain $\left(\mathrm{NS} 114^{\mathrm{T}}\right)$. Stand Genomic Sci 2009; 1:133-140. doi:10.4056/sigs.19262

14. Raj HD. 1981. The genus Microcyclus and related bacteria, p. 630-44. In: Starr MP, Stolp H, Trüper HG, Balows A, HG Schlegel (eds), The Prokaryotes. A handbook on habitats, isolation and identification of bacteria, Vol 1. Springer, New York.

15. Raj HD, Maloy SR. Proposal of Cyclobacterium marinus gen. nov., comb. nov. for a marine bacterium previously assigned to the genus Flectobacillus. Int J Syst Bacteriol 1990; 40:337-347. doi:10.1099/00207713-40-4-337

16. Field D, Garrity G, Gray T, Morrison N, Selengut J, Sterk P, Tatusova T, Thomson N, Allen MJ, Angiuoli SV, et al. The minimum information about a genome sequence (MIGS) specification. Nat Biotechnol 2008; 26:541-547. PubMed doi:10.1038/nbt1360
17. Woese CR, Kandler O, Wheelis ML. Towards a natural system of organisms: proposal for the domains Archaea, Bacteria, and Eucarya. Proc Natl Acad Sci USA 1990; 87:4576-4579. PubMed doi:10.1073/pnas.87.12.4576

18. Garrity GM, Lilburn TG, Cole JR, Harrison SH, Euzéby J, Tindall BJ. Taxonomic outline of the Bacteria and Archaea, Release 7.7 March 6, 2007. Part 11 - The Bacteria: Phyla "Planctomycetes", "Chlamydiae", "Spirochaetes", "Fibrobacteres", "Acidobacteria", "Bacteroidetes", "Fusobacteria", "Verrucomicrobia", "Dictyoglomi", "Gemmatimonadetes", and "Lentisphaerae". http://www.taxonomicoutline.org.

19. Woese CR, Stackebrandt E, Macke TJ, Fox GE. A phylogenetic definition of the major eubacterial taxa. Syst Appl Microbiol 1985; 6:143-151. PubMed

20. Garrity GM, Holt JG. In: Garrity GM, Boone DR, Castenholz RW (2001) Taxonomic Outline of the Archaea and Bacteria. Bergey's Manual of Systematic Bacteriology 155-166.

21. Stanier RY. Studies on the Cytophagas. J Bacteriol 1940; 40:619-635. PubMed

22. Larkin JM, Williams PM, Taylor R. Taxonomy of the genus Microcyclus Orskov 1928: reintroduction and emendation of the genus Spirosoma Migula 1894 and proposal of a new genus, Flectobacillus. Int J Syst Bacteriol 1977; 27:147-156. doi:10.1099/00207713-27-2-147

23. Classification of Bacteria and Archaea in risk groups. www.baua.de TRBA 466.

24. Ashburner M, Ball CA, Blake JA, Botstein D, Butler $\mathrm{H}$, Cherry JM, Davis AP, Dolinski K, Dwight SS, Eppig JT, et al. Gene Ontology: tool for the unification of biology. Nat Genet 2000; 25:25-29. PubMed doi:10.1038/75556

25. Gordon RE, Mihm JM. A comparative study of some strains received as nocardiae. / Bacteriol 1957; 73:15-27. PubMed

26. Urakami T, Komagata K. Methanol-utilizing Ancylobacter strains and comparison of their cellular fatty acid compositions and quinone systems with those of Spirosoma, Flectobacillus, and Runella species. Int J Syst Bacteriol 1986; 36:415-421. doi:10.1099/00207713-36-3-415

27. List of growth media used at DSMZ: http://www.dsmz.de/microorganisms/media list.p $\underline{\mathrm{hp}}$

28. Wu D, Hugenholtz P, Mavromatis K, Pukall R, Dalin E, Ivanova NN, Kunin V, Goodwin L, Wu 
$\mathrm{M}$, Tindall BJ, et al. A phylogeny-driven genomic encyclopedia of Bacteria and Archaea. Nature 2009; 462:1056-1060. PubMed doi:10.1038/nature08656

29. Sims D, Brettin T, Detter J, Han C, Lapidus A, Copeland A, Glavina Del Rio T, Nolan M, Chen F, Lucas $\mathrm{S}$, et al. Complete genome sequence of $\mathrm{Ky}$ tococcus sedentarius type strain $\left(541^{\mathrm{T}}\right)$. Stand Genomic Sci 2009; 1:12-20. doi:10.4056/sigs.761

30. Hyatt $\mathrm{D}$, Chen GL, Locascio PF, Land ML, Larimer FW, Hauser LJ. Prodigal Prokaryotic Dynamic Programming Genefinding Algorithm. BMC
Bioinformatics 2010; 11:119. PubMed

doi:10.1186/1471-2105-11-119

31. Pati A, Ivanova N, Mikhailova N, Ovchinikova G, Hooper SD, Lykidis A, Kyrpides NC. GenePRIMP: A Gene Prediction Improvement Pipeline for microbial genomes. Nat Methods (in press).

32. Markowitz VM, Mavromatis K, Ivanova NN, Chen IMA, Chu K, Kyrpides NC. Expert Review of Functional Annotations for Microbial Genomes. Bioinformatics 2009; 25:2271-2278. PubMed doi:10.1093/bioinformatics/btp393 